\title{
Hyperspectral Response of Rice Canopy and Prediction of Its Chlorophyll Content in Cold Regions
}

\author{
Jianqing Yuan ${ }^{1,2}$, Zhongbin $\mathrm{Su}^{1 *}$, Qingming Kong ${ }^{1}$, Li Kang ${ }^{1,3}$, Qi Zhang ${ }^{1}$ and \\ Yu Zhang ${ }^{1}$ \\ ${ }^{1}$ College of Electrical and Information, Northeast Agricultural University, Harbin, \\ 150030, China \\ ${ }^{2}$ Faculty of Electrical Engineering and Computer Science, East University of \\ Heilongjiang, Harbin, 150066, china \\ ${ }^{3}$ School of Information Science and Engineering, Dalian Polytechnic University, \\ Dalian, 116034, china \\ E-mail:yuanyuanjq@126.com
}

\begin{abstract}
Based on field trials of different nitrogen levels, rice canopy chlorophyll content and canopy spectrum of its booting stage were respectively measured using the nitrogen balance index meter and the hyperspectral imager, in the Northeast of china. Analyzing the rice canopy spectral response characteristics under nitrogen gradient after canopy reflectance spectra were first extracted and filtered for noise, then the original reflectance spectra were transformed into the first derivative reflectance for further analysis. Stepwise analysis was carried out to select sensitive bands for canopy chlorophyll content by making correlation analysis and to build spectral indexes, and then chlorophyll content prediction models were generated and tested. After comparing, the two models with better estimation precision were the model with reflectivity at $572 \mathrm{~nm}$ as a variable and the model with the index DSI (572,682) constructed the reflectivity at 572 and $682 \mathrm{~nm}$ as a variable, the coefficients of determination $R^{2}$ were 0.733 and 0.694 , the root mean square errors RMSE were 2.9656 and 3.0346, and the relative errors $R E \%$ were $7.07 \%$ and $7.43 \%$.
\end{abstract}

Keywords: rice, canopy spectrum, canopy chlorophyll content, prediction model

\section{Introduction}

Chlorophyll is an important indicating factor of crop physiology and its growth status, canopy chlorophyll content can effectively reflects population characteristics and growing status of crops [1-3]. The study has shown that hyperspectral remote sensing technology can quickly and efficiently estimate canopy chlorophyll content of crops in the canopy level, and then monitor their nutritional and growing status [4-7].

Hyperspectral imaging technique is a new non-destructive testing way integrating spectroscopy and imaging technology, compared to the non-imaging, it provides all information of the morphological features, the internal structural characteristics and the chemical composition characteristics to the measured object [8-9]. Many ways are used to select spectral parameters when building prediction models, while some spectral parameters is invalid in the late model validation. To avoid doing so, it is very important to select the spectral parameters effectively.

In the northeast of china, the booting stage of rice is the critical period of growth and development, in this period rice is sensitive to water and fertilizer and finally affect grain quality and yield [10]. For this reason, in the booting stage of rice, the hyperspectral images were captured by hyperspectral imager (Headwall) and canopy chlorophyll 
content was measured using nitrogen balance index meter (Dualex). Canopy chlorophyll prediction models were established on the basis of hyperspectral technology after selecting appropriately multiple spectral parameters, and then the prediction models were validated and evaluated, which will provide technical support for the management of nitrogen in rice field.

\section{Materials and Methods}

\subsection{Study Area}

The study site was located FangZheng Rice Research Institute in Harbin, nitrogen fertilizer was different gradient processing, water, potash and phosphate was the standard management. Nitrogen was set to six levels (N0, N1, N2, N3, N4, N5), fertilizer were separately $0,50,70,100,130,150$ and $\mathrm{N} 3$ was normal fertilization. Fertilizer was respectively applied into each unit in basal fertilizer, turning green and booting stage by $40 \%, 30 \%$ and $30 \%$. There were two species of rice in trial, each species was treated repeatedly four times, that is, each species has a total of 24 units, and each unit was $10 \mathrm{~m} * 10 \mathrm{~m}$, protection line was the plastic film mud mounds to separating from each unit.

\subsection{Hyperspectral Measurements and Pre-processing}

At 11:00 on July 13th, 2014, rice canopy hyperspectral images of booting stage were captured using Headwall in FangZheng Rice Research Institute. The slit width of Hyperspectral imager is $25 \mathrm{pm}$, spectral range was $400 \sim 1000 \mathrm{~nm}$, spectral resolution is $2.4 \mathrm{~nm}$, band interval is set to $3 \mathrm{~nm}$ when capturing images. Its sensor imaging modality is linear push-broom, it was mobile pushed broom by placed on $2 \mathrm{~m}$-long rail which was from the ground $2 \mathrm{~m}$, width of visual field is $1.2 \mathrm{~m}$, the spatial resolution is $0.25 \mathrm{~mm}$. And standard white calibration is performed before each sample.

Using ENVI software to process atmospheric and geometric correction and to extract spectral curves, extracting randomly spectral curves of six points in the center of each unit, their average value was treated as canopy spectra of this district, 48 extracted canopy spectra samples were exported. Because spectrums between 920nm 1000nm were disturbed seriously by instrument noise and their curves become jagged, these bands were removed, then a total of 176 bands of data were retained. And we use Savitzky-Golay method to remove noise and filter spectral data, smoothing point was 4 .

Research shows that the differential technology can reduce or eliminate the effects of soil and other basic background on canopy spectra, so rice canopy spectrum was treated using differential techniques in this study. Because the spectrum acquired was discrete, thus the first-order derivative of the formula is for:

$$
D=\frac{R_{i+1}-R_{i-1}}{\left(\rho_{i+1}-\rho_{i}\right)+\left(\rho_{i}-\rho_{i-1}\right)}=\frac{R_{i+1}-R_{i-1}}{2 \Delta \rho}
$$

Where, $\mathrm{D}$ is the first derivative of the band $\mathrm{i}, \rho_{i}$ is the wavelength.

\subsection{Canopy Chlorophyll Measurements}

At the same time, canopy chlorophyll content was measured using nitrogen balance index instrument (Dualex), which was through the transmittance of the light to quickly measure the chlorophyll content of leaves. Six plants of growing uniform were selected to be measured in unit center, all the blades in the top of chosen plants were measured and measurements were repeated five times in the middle position of each blade. Finally, the average of all six plants chlorophyll content was look upon as the canopy chlorophyll content of the unit, so 48 samples were acquired. 


\subsection{Data Analysis}

Correlation analysis was respectively performed between the canopy chlorophyll content and the original spectrum, the first derivative spectra, the correlation coefficient graphs were plotted, the sensitive bands were determined. Basis of these sensitive bands, structuring the original spectral reflectivity $(\mathrm{R})$, the first differential reflectivity(R') and common vegetation index: the ratio index(RSI), the difference index(DSI), the normalized difference index(NDSI) [11], plus chlorophyll index LCI [12] to analyze their relationship with the rice canopy chlorophyll content, and ultimately to build predictive models. Each index formula is as follows:

$$
\begin{aligned}
L C I & =\left(R_{850}-R_{710}\right) /\left(R_{850}-R_{680}\right) \\
R S I & =\left(R_{\rho 1}, R_{\rho 2}\right)=R_{\rho 1} / R_{\rho 2} \\
D S I & =\left(R_{\rho 1}, R_{\rho 2}\right)=R_{\rho 1}-R_{\rho 2} \\
N D S I & =\left(R_{\rho 1}, R_{\rho 2}\right)=\left(R_{\rho 1}-R_{\rho 2}\right) /\left(R_{\rho 1}+R_{\rho 2}\right)
\end{aligned}
$$

Where, $R_{\rho 1}$ and $R_{\rho 2}$ is respectively for each band spectral reflectance.

The 48 samples collected were divided into two groups, 32 samples were randomly selected for modeling and the remaining 16 samples for testing the model. Accuracy of the model was checked and evaluated by the correlation coefficient (R2), root mean square error RMSE (Equation 5) and relative error RE\% (Equation 6), finally diagrams of 1:1 relationship between measured and predicted values were draw. $\hat{y}_{i}$ and $y_{i}$ is respectively the predicted and the measured value of chlorophyll.

$$
\begin{aligned}
& R M S E=\sqrt{\frac{\sum_{i=1}^{n}\left(\hat{y}_{i}-y_{i}\right)^{2}}{n}} \\
& R E \%=\frac{1}{n} \sum_{i=1}^{n} \frac{\left|\hat{y}_{i}-y_{i}\right|}{y_{i}} \times 100 \%
\end{aligned}
$$

Hyperspectral pre-processing and data analysis were performed in Excel and Matlab.

\section{Results and Discussion}

\subsection{Spectral Response of Rice Canopy Chlorophyll under Different Nitrogen Levels}

To obtain the changes of spectral reflectivity under six different nitrogen levels, taking the average of reflectance spectrum for each rice varieties in the same nitrogen level of the four units repeated. In order to facilitate the display, reflectance spectra curves of each rice species were respectively drawn only at N0, N3 and N5 nitrogen levels, shown in Figure 1.

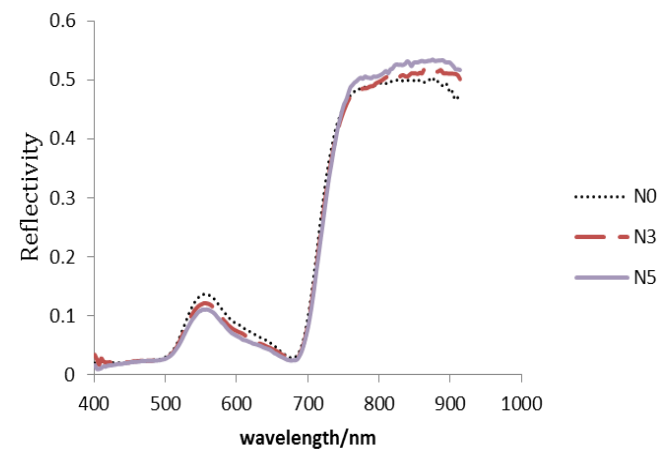




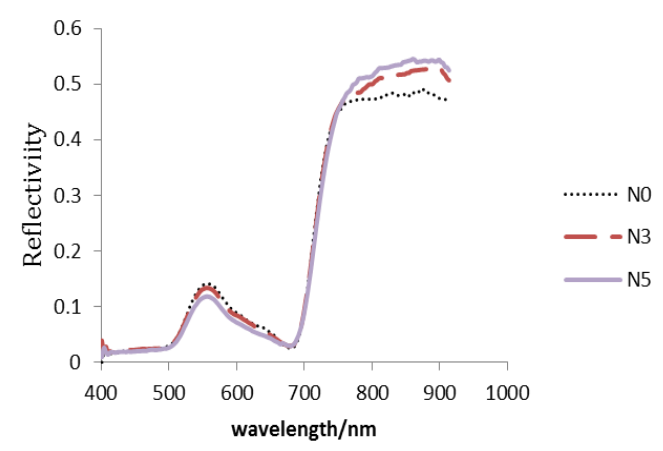

\section{Figure 1. Reflectivity Spectrum of Rice Canopy under Different Nitrogen} Levels at Booting Stage

As can be seen from Figure 1, in booting stage, canopy reflectance spectra of two rice varieties show the same changes at different nitrogen levels: with the increasing nitrogen levels, the reflectivity decreases in the visible region and increases in near infrared region, because canopy chlorophyll content increases with the larger amount nitrogen.

\subsection{Correlation Analysis}

Figure 2 is the first derivative of the original spectrum graph, it shows that the reflectance point increasing fastest is $718 \mathrm{~nm}$ (located at red edge position) in the visible between $680-740 \mathrm{~nm}$, which is the inflection point of the first derivative spectrum, and the other three more obvious inflection are located in the $525 \mathrm{~nm}, 572 \mathrm{~nm}$ and $655 \mathrm{~nm}$.

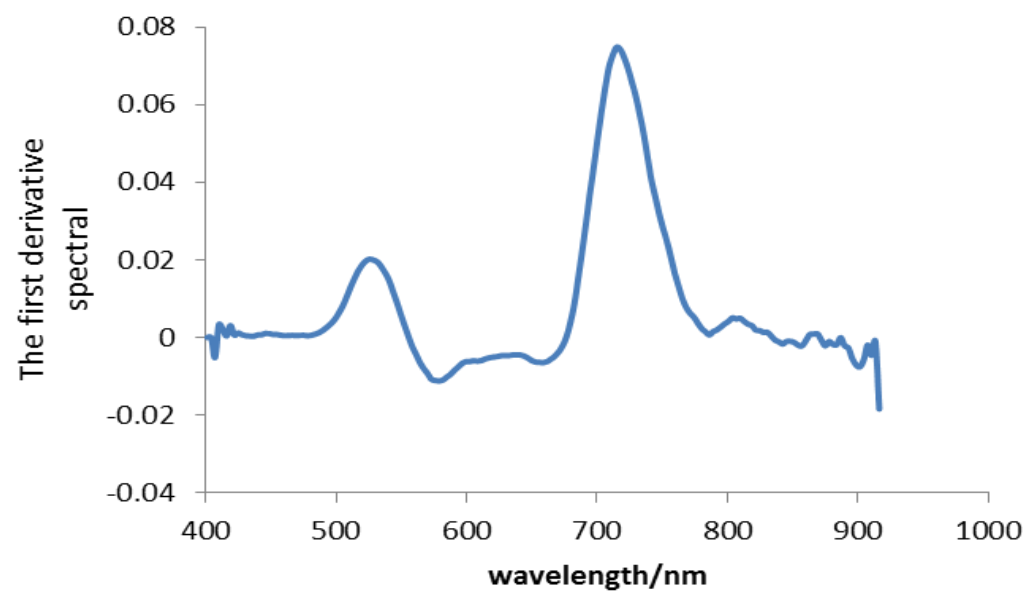

Figure 2. Curve of the First Derivative Spectral Data

Figure 3 is respectively the related curves of rice canopy chlorophyll content with original spectrum and of rice canopy chlorophyll content with the first-order derivative spectra in rice booting stage. As can be seen from the Figure, the correlation between rice canopy chlorophyll content and the original spectrum is significant in band 460 740nm, showing a significant negative correlation, there is a peak at $682 \mathrm{~nm}$ and its correlation coefficient is -0.60046 , which divides this range into two parts: correlation coefficients are $-0.7790 \sim-0.8238$ in $510 \sim 660 \mathrm{~nm}$, correlation coefficients are $0.7364-0.8025$ in 694 $\sim 735 \mathrm{~nm}$. The correlation of rice canopy chlorophyll content with the first derivative spectra reflects significantly in five band regions, showing negative correlation at the 500 $\sim 550,685 \sim 715 \mathrm{~nm}$ and positive correlation at $570 \sim 600,640 \sim 670$ and $730 \sim 740 \mathrm{~nm}$ place. 

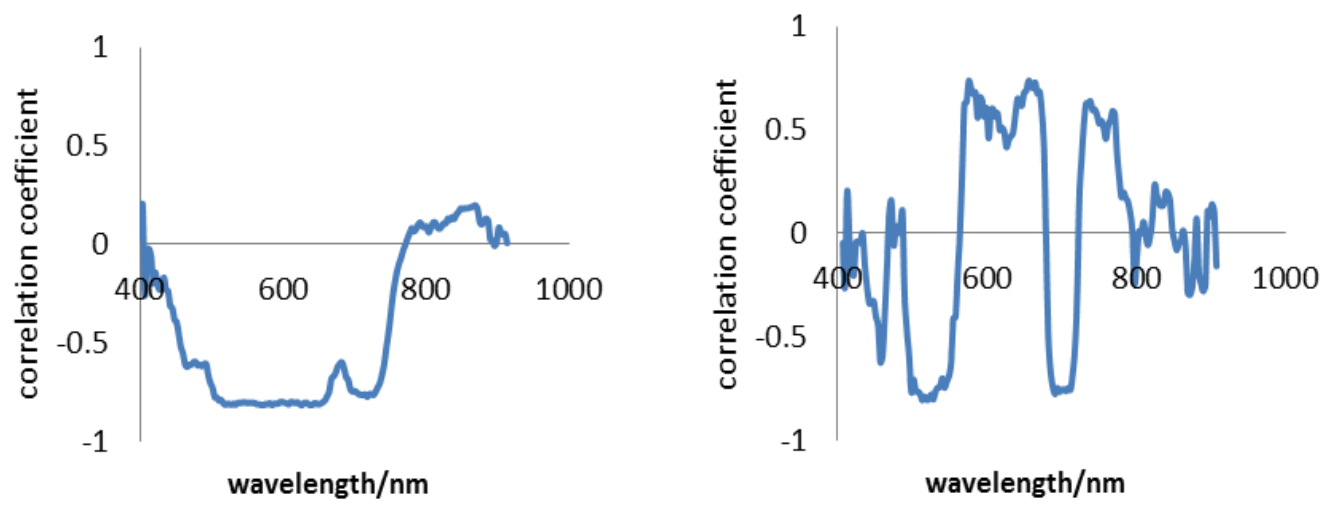

Figure 3. Correlation Coefficients of Original Spectral Data and its First Derivative Spectral Data with Canopy Chlorophyll Content

\subsection{Modelling Canopy Chlorophyll}

Based on sensitive bands area and the first-order differential spectra of each inflection point, selecting the original reflectance spectrum $(572 \mathrm{~nm}$ and $718 \mathrm{~nm}$ nearby) at which the correlation coefficients with rice canopy chlorophyll not only are reached the largest respectively but also are inflection points of the first derivative, and combining with spectral reflectance of the peak (682 nm near) between the two spectral troughs to construct the difference parameter (DSI), the ratio parameter (RSI) and the normalization parameter (NDSI); in the same way, to build relevant parameters choosing the maximum correlation coefficients respectively in three sensitive areas where canopy chlorophyll and the first derivative spectral reflectance relate significantly.

Calculating chlorophyll index LCI, the correlation coefficient is 0.7612 , so it is also as a sensitive parameter. The correlation coefficients of canopy chlorophyll with these parameters are given in Table 1.

Table 1. Correlation Coefficient between Spectral Parameters and Canopy Chlorophyll Content

\begin{tabular}{|c|c|c|c|}
\hline spectral parameter & correlation coefficient & spectral parameter & correlation coefficient \\
\hline LCI & $0.7612^{* *}$ & $\mathrm{R}_{513}^{\prime}$ & $-0.8114^{* * *}$ \\
\hline $\mathrm{R}_{572}$ & $-0.8238^{* *}$ & $\mathrm{R}_{655}^{\prime}$ & $0.7511^{* *}$ \\
\hline $\mathrm{R}_{718}$ & $-0.8025^{* *}$ & $\mathrm{R}_{712}^{\prime}$ & $-0.7933^{* *}$ \\
\hline $\operatorname{RSI}(572,682)$ & $-0.4785^{* *}$ & $\operatorname{RSI}\left(\mathrm{R}_{513}^{\prime}, \mathrm{R}_{655}{ }_{65}\right)$ & -0.4101 \\
\hline $\operatorname{RSI}(718,572)$ & $0.7382^{* *}$ & $\operatorname{RSI}\left(\mathrm{R}^{\prime}{ }_{712}, \mathrm{R}^{\prime}{ }_{513}\right)$ & $0.71352^{* *}$ \\
\hline $\operatorname{RSI}(718,682)$ & -0.0562 & $\operatorname{RSI}\left(\mathrm{R}_{712}^{\prime}, \mathrm{R}_{655}{ }_{65}\right)$ & $-0.6312^{* *}$ \\
\hline $\operatorname{DSI}(572,682)$ & $-0.8282^{* *}$ & $\operatorname{DSI}\left(\mathrm{R}_{513}{ }_{5}, \mathrm{R}_{655}{ }_{65}\right)$ & $-0.8210^{* *}$ \\
\hline $\operatorname{DSI}(718,572)$ & $-0.7059^{* *}$ & $\operatorname{DSI}\left(\mathrm{R}_{712}{ }_{71}, \mathrm{R}^{\prime}{ }_{513}\right)$ & $-0.7279^{* *}$ \\
\hline $\operatorname{DSI}(718,682)$ & $-0.7983^{* *}$ & $\operatorname{DSI}\left(\mathrm{R}_{712}{ }_{712} \mathrm{R}_{655}\right)$ & $-0.807^{* *}$ \\
\hline $\operatorname{NDSI}(572,682)$ & $-0.4776^{* *}$ & $\operatorname{NDVI}\left(\mathrm{R}_{513}{ }_{51}, \mathrm{R}_{655}{ }_{65}\right)$ & -0.4094 \\
\hline $\operatorname{NDSI}(718,572)$ & $0.7391^{* *}$ & $\operatorname{NDVI}\left(\mathrm{R}^{\prime}{ }_{712}, \mathrm{R}^{\prime}{ }_{513}\right)$ & $0.7141^{* *}$ \\
\hline $\operatorname{NDSI}(718,682)$ & -0.0437 & $\operatorname{NDVI}\left(\mathrm{R}^{\prime}{ }_{712}, \mathrm{R}_{655}{ }_{65}\right)$ & $-0.6564^{* *}$ \\
\hline
\end{tabular}

$* * \mathrm{p}<0.01$ The same below 
The study selects five spectral parameters that the absolute value of correlation coefficients were above 0.8 depending on the size of the correlation coefficient, these five spectral parameters were: $\mathrm{R}_{572}, \mathrm{DSI}(572,682), \mathrm{R}_{513}, \operatorname{DSI}\left(\mathrm{R}_{712}, \mathrm{R}_{655}{ }_{65}\right)$ and $\mathrm{R}_{718}$. The prediction models of rice canopy chlorophyll were constructed in the forms of linear, logarithmic, quadratic polynomial, power and exponential, based on the above five parameters plus chlorophyll index LCI. Six predicting models were generated according to the value size of $\mathrm{R}^{2}$, which are given in Figure 4, showing that the models of six parameters all reach a significant level 0.01 .
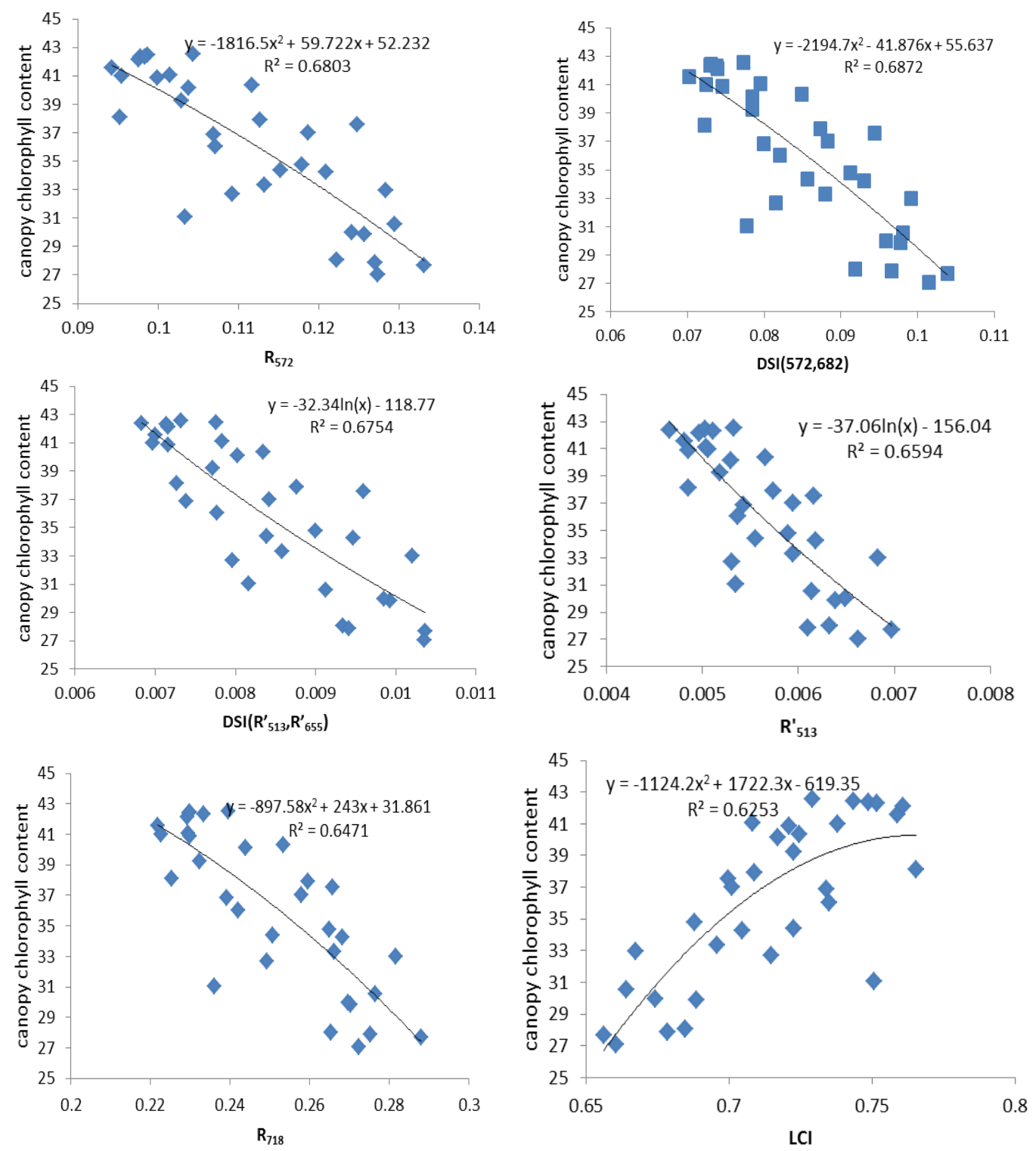

Figure 4. Prediction Model of Canopy Chlorophyll Content based on Spectral Parameters

\subsection{Model Validation}

The six prediction models filtered out are tested and validated using 16 data of canopy chlorophyll content and their spectral reflectivity, the testing results are shown in table 2. 
Table 2. Fitting Accuracy Parameters of the Model Checking

\begin{tabular}{lrccc}
\hline \multicolumn{1}{c}{ value } & fitting equation & $\mathrm{R}^{2}$ & $\mathrm{RMSE}$ & $\mathrm{RE} \%$ \\
\hline $\operatorname{DSI}(572,682)$ & $\mathrm{y}=0.617 \mathrm{x}+12.42$ & $0.694^{* *}$ & 3.0346 & 7.43 \\
$\mathrm{R}_{572}$ & $\mathrm{y}=0.669 \mathrm{x}+10.35$ & $0.733^{* *}$ & 2.9656 & 7.07 \\
$\mathrm{R}_{718}$ & $\mathrm{y}=0.564 \mathrm{x}+14.48$ & $0.612^{* *}$ & 3.2708 & 7.97 \\
DSI $\left.^{*} \mathrm{R}^{\prime *}{ }_{513}, \mathrm{R}^{\prime}{ }_{655}\right)$ & $\mathrm{y}=0.586 \mathrm{x}+13.16$ & $0.674^{* *}$ & 3.2964 & 7.90 \\
$\mathrm{R}^{\prime}{ }_{513}$ & $\mathrm{y}=0.581 \mathrm{x}+13.11$ & $0.735^{* *}$ & 3.2830 & 7.86 \\
$\mathrm{LCI}$ & $\mathrm{y}=0.678 \mathrm{x}+12.35$ & $0.490^{* *}$ & 4.0250 & 9.75 \\
\hline
\end{tabular}

From Table 2, we can see the five models have higher fitting precision except for the model with LCI as a variable. Among them, the model with the reflectivity at $572 \mathrm{~nm}$ as a variable is the best fitting, the coefficient of determination $\mathrm{R}^{2}$ is 0.733 , the root mean square error RMSE is 2.9656, and the relative error RE\% is $7.07 \%$; the followed better is the model with the index DSI $(572,682)$ constructed the reflectivity at 572 and $682 \mathrm{~nm}$ as a variable, the coefficient of determination $\mathrm{R}^{2}$ is 0.694 , the root mean square error RMSE is 3.0346 , and the relative error RE\% is $7.43 \%$. The curves showing estimation accuracy of two predictive models are given in Figure 5: the left is with the spectral reflectance at $572 \mathrm{~nm}$ as a variable, the right is with the DSI $(572,682)$ as a variable (thick line is 1: 1 line).
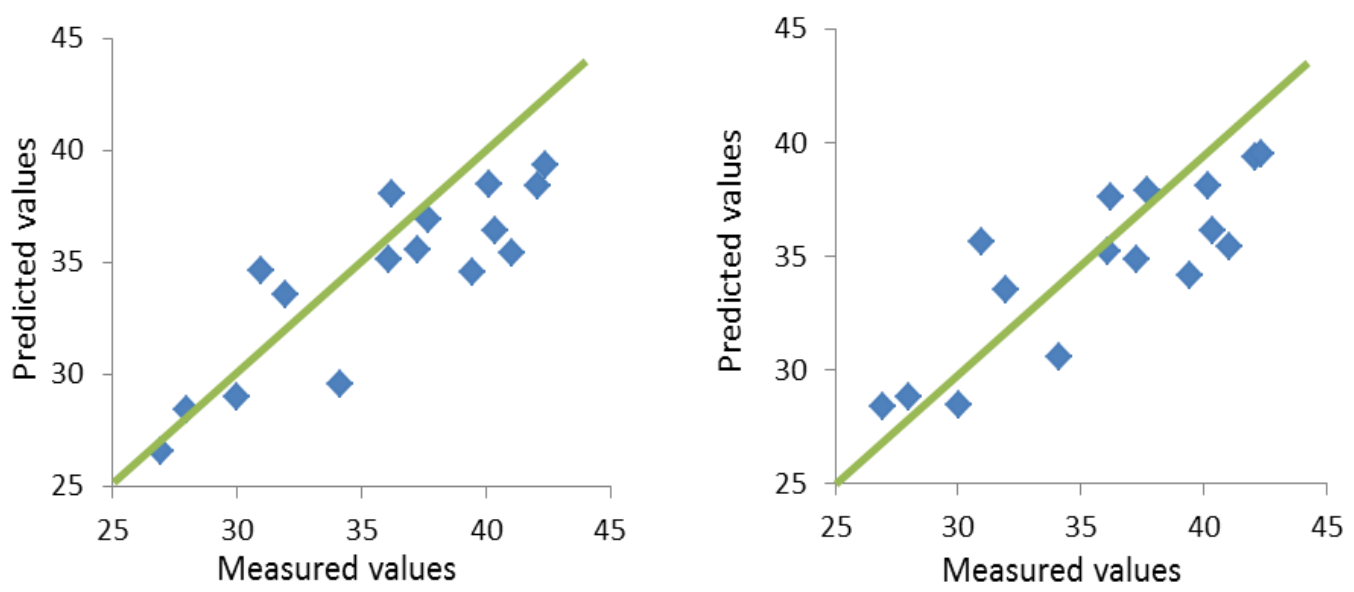

Figure 5. Relationship between Measured and Predicted Values of Canopy Chlorophyll Content

\section{Conclusions}

Based on field trials, the study discussed the rice canopy spectral response characteristics under different nitrogen levels on a critical growth period (the booting stage) in the Northeast of china, then build and tested models through determining the sensitive bands of hyperspectral response after transforming the first-order derivative spectra and making the correlation analysis. The following conclusion could be made from the present research:

(1) Two rice varieties under different nitrogen levels show the same changes difference: with the increasing nitrogen levels, the reflectivity decreases in the visible region and increases in near infrared region.

(2) In the Northeast of china, hyperspectral sensitive bands of rice canopy chlorophyll in booting stage were mainly concentrated in the visible band and the red edge position, there were some sensitive band point be found in 460 740nm. 
(3) Rice canopy chlorophyll prediction models were generated: the model with the reflectivity at $572 \mathrm{~nm}$ as a variable $(\mathrm{y}=-1816.5 \mathrm{x} 2+59.722 \mathrm{x}+52.232)$, and the model with the difference index $\operatorname{DSI}(572,682)$ as a variable $(y=-2194.7 x 2-41.876 \mathrm{x}+55.637)$. The two predicting models have better prediction precision upon examination.

The present investigation was carried out based on the data collected during a single growth stage. Further research will be to develop a more robust model for estimation of rice canopy chlorophyll by increasing the number of sampling at different growing period and sites.

\section{Acknowledgements}

The authors would like to acknowledge the National High-tech R\&D Program of China (863 Program)(2013AA102303) and the Key Technologies R\&D Program of Harbin (2013AA6BN010).

\section{References}

[1] W. Fumin, H. Jingfen, L. Zhanyu, et al., "Optimal simple ratio pigment index for estimating pigment contents of rice", Journal of Zhejiang University: Agric And Life Sci, vol. 3, no. 35, (2009).

[2] Y. Jinguo and N. Zheng, "Nitrogen and chlorophyll mapping based on Hyperion hyperspectral image", Transactions of the Chinese Society of Agricultural Engineering, vol. 4, no. 23, (2007).

[3] Y. Feng, F. Yamin, L. Jialong, et al., "Estimating LAI and CCD of rice and wheat using hyperspectral remote sensing data", Transactions of the Chinese Society of Agricultural Engineering, vol. 2, no. 26, (2010).

[4] X. H. Yang, J. F. Huang, Y. P. Wu, et al., "Estimating biophysical parameters of rice with remote sensing data using support vector machines", Science China (Life Science), vol. 3, no. 54, (2011).

[5] J. F. Huang and G. A. Blackburn, "Optimizing predictive models for leaf chlorophyll concentration based on continuous wavelet analysis of hyperspectral data", International Journal of Remote Sensing, vol. 24, no. 32, (2011).

[6] W. M. Mark, B. Stewart and L. Robertson, "An algorithm for detecting trophic status (chlorophyll-a), cyanobacterial-dominance, surface scrums and floating vegetation in inland and coastal waters", Remote Sensing of Environment, vol. 1, no. 24, (2012).

[7] G. V. Luis, S. Evangelos, J. M. T. Palenzuela, "Neural network estimation of chlorophyll a from MERIS full resolution data for the coastal waters of Galioian rias (NW Spain)", Remote Sensing of Environment, vol. 2, no. 115, (2011).

[8] R. Lu, "Nondestructive measurement of firmness and soluble solids content for apple fruit using hyperspectral scattering images", Sensing and Instrumentation for Food Quality and safety, vol. 1, no. 1, (2007).

[9] H. Min and Z. Qi-Bing, "Feature extraction of hyperspectral scattering image for apple mealiness based on singular value decomposition”, Spectroscopy and Spectral Analysis, vol. 3, no. 31, (2011).

[10] L. Xiuhua and L. Minzan, "Monitoring of rice plant growth in Northeast China using dual-wavebands crop growth analyzer", Transactions of the Chinese Society of Agricultural Engineering, vol. 8, no. 27, (2011).

[11] C. Zhi-Qiang, W. Lei and B. You-Lu, "Spectral Response of Maize Leaves and Prediction of Their Nitrogen Content", Spectroscopy and Spectral Analysis, vol. 4, no. 33, (2013).

[12] B. Datt, "Visible/near infrared reflectance and chlorophyll content in Eucalyptus leaves", International Journal of Remote Sensing, vol. 14, no. 20, (1999).

\section{Author}

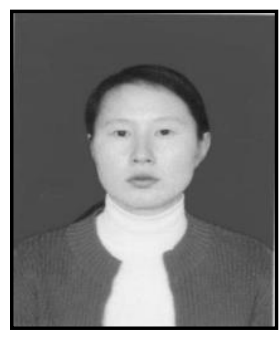

Jianqing Yuan, Born in 1979, female, associate professor, master, she is engaged in research of hyperspectral remote sensing. Email:yuanyuanjq@126.com, Tel:13936640187. 